\title{
A study of collaboration via a digital space accessible to adolescents with visual impairment
}

\author{
Aurélie Vallée ${ }^{1}$, Katia Rovira ${ }^{1}$ \& Loïc Deschamps ${ }^{2}$ \\ 1 Université de Rouen, France \\ 2 Université de Technologie de Compiègne, France
}

\begin{abstract}
Corresponding author:
Aurélie Vallée, Laboratoire PSY.NCA (EA 4700), Université de Rouen, 76821 Mont Saint Aignan Cedex, France.

Email:valleaur@gmail.com

To cite this article:

Vallée, A., Rovira, K. \& Deschamps, L., (2016). A study of collaboration via a digital space accessible to adolescents with visual impairment. British Journal of Visual Impairment. January 2016, 34: 83-90, doi:10.1177/0264619615612525
\end{abstract}

\section{Abstract}

The aim of this study is to observe whether the improvement of tactile digital space allows the visually impaired adolescents to interact and collaborate. A total of 12 participants (average age: 14 years 9 months) have to interact in pairs through a tactile device and have to solve a common task in a shared digital space. Contextualization of the environment and two sorts of tactile stimulation are proposed to the participants. The results and a semi-structured interview show that the subjects were able to set up an effective collaboration (strategies for following each other), which were facilitated by tactile feedback incorporating a degree of "parallelism" which makes it possible to perceive the direction of the movement of the other subject.

\section{Keywords}

Adolescents, collaboration, educational support, sensory substitution, visual impairment

\section{Introduction}

In recent years, a number of sensory substitution devices have been built in order to allow visually impaired persons to have access to graphical digital information. For example, the tactile mouse VTPlayer ${ }^{\mathrm{TM}}$ makes it possible to explore digital geographical maps (Jansson, Juhasz, \& Cammilton,2006). On the same principle, the Cognitive Research and Enaction Design (CRED) team at the Technological University of Compiegne has developed the Tactos device. This system makes it possible to transpose the visual information displayed on a computer screen into tactile information using a matrix of tactile stimulators (Figure 1). 

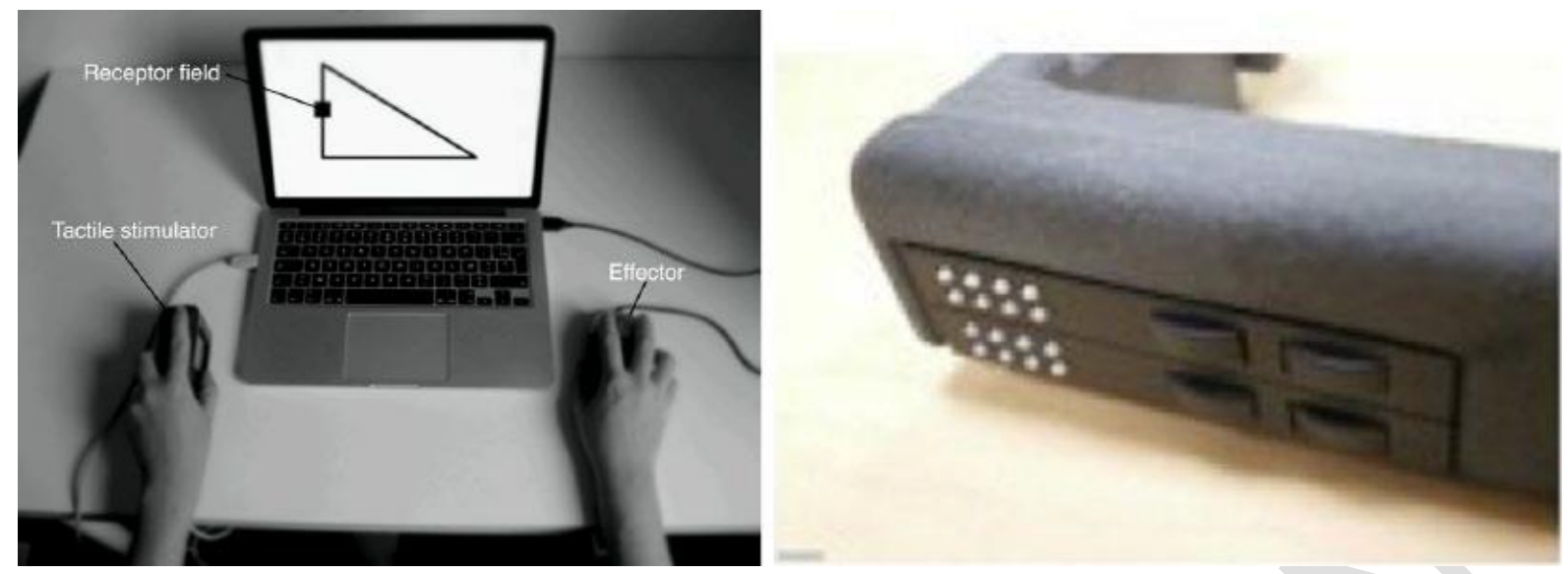

Figure 1 : Tactos device

The user moves a cursor with 16 receptor fields by means of an effector (i.e., a mouse). When one or more of these receptor fields encounter a black pixel on the screen, the corresponding tactile stimulators are activated (Figure 2).

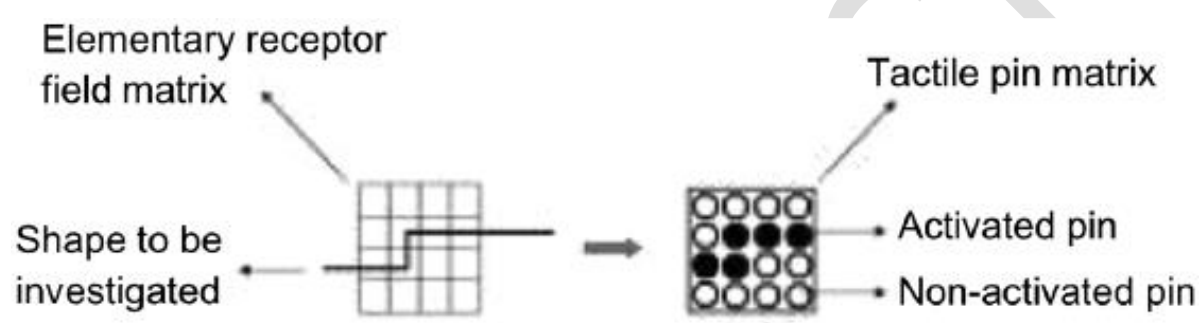

Figure 2 : Tactile stimulators

The user manipulates the effector with her dominant hand, and the tactile stimulators are placed under the fingers of the free hand.

Several studies have shown that Tactos makes it possible to locate and recognize digitalized graphical contents (icons, geometric shapes, geographical maps, etc.) (Sribunruangrit, Marque, Lenay, Gapenne, \& Vanhoutte, 2002, 2004). Within the context of school, Rovira and Gapenne (2009) have carried out a longitudinal study of three visually impaired adolescents. The authors observed that these Tactos users were able to explore, identify, and categorize geometric shapes. In line with these results, Tactos can be considered as a relevant educational tool (Rovira, Gapenne, \& Vallée, 2014).

Computer technologies are taking an increasingly important role in education. Most of them give pride of place to communication, in order to favor collaborative learning between students. Indeed, systems involving Computer-Supported Collaborative Learning (CSCL) (loannidou et al.,2010) are proved to facilitate knowledge constitution and sharing through the collaborative interactions between geographically distant users (e.g., Resta \& Laferrière, 2007).

On the model of the CSCL, Tactos' designers have created a shared digital space called Intertact (designed by D. Aubert): it consists in connecting several Tactos devices through the network, thus allowing for tactile encounters between users. Each user controls the movements of an avatar (corresponding to the cursor used in the Tactos device) that the other user can perceive by means of their own avatar (i.e., the superposition of the avatars triggers a simultaneous stimulus for both users). Thus, this device can be considered as a basis for interaction and collaboration, in which two or more users (with or without visual impairment) can share a pedagogical content. 
However, for an effective collaboration, it seems necessary that participants are aware of sharing a common goal (Fiebich \& Gallagher, 2013). It is also important that their activities take place into specific context in order to make the intentions' perception of both easier (Searle, 1990). The framework of CSCL actually proposes graphic representations of the environment and if appropriate, of the others as avatars, allowing localization, identification, and representation of the attentional focus of the other (Capin, Pandzic, Thalmann, \& Thalmann, 1997). As a particular form of CSCL, Intertact gives a tactile access to the environment and the localization of the avatars. In order to design a meaningful environment, and to promote collaboration with appropriate tactile feedbacks, preliminary studies of tactile interaction have been carried out with sighted adults in one-dimensional minimalist

environments. First, they were able to coordinate the movements of their avatars, which led to an emergent collective dynamics, sustaining the interaction process over time. That coordination was proved to support the recognition of the other (Auvray, Lenay, \& Stewart, 2009). Second, Deschamps (2013) investigated whether the adults could decide, from two static objects, which was the one that was also perceptible by their partner. Three types of tactile feedbacks were manipulated there. The results showed that sighted adults did succeed in the task, whatever the type of tactile feedbacks, even if the latter influenced the way they performed it.

The question of the relevance of Intertact for education convinced us to pursue this line of research with younger, visually impaired participants. As a first attempt, we replicated the protocol of Deschamps (2013) in visually impaired adolescents (Vallée, Deschamps, \& Rovira, 2012). The results showed that $42.8 \%$ of the adolescents ( $N=$ 14) did not succeed in the task. Following their own statements, the lack of contextualization of the environment and the absence of concomitant feedbacks concerning their performance were harmful.

We present here an extension of this study, contextualizing the task and the environment with fantasy-like features, and introducing specific feedbacks to inform the participants whether they had succeeded in the different trials or not.

The aim of this study is to observe to what extent the contextualization of the task will promote the interaction and collaboration between visually impaired participants and to explore the effects of two types of tactile stimuli upon that collaboration.

\section{Method}

\section{Subjects}

A total of 12 visually impaired adolescents participated in this study. Participants ranged from 11 to 16 years in age, with the majority over the age of 13 ( $M=14.9$, standard deviation $[S D]=1.65$ ) years. The male and female proportions were $66.66 \%$ and $33.33 \%$, respectively.

For reasons of privacy and confidentiality, we did not have access to their medical files. We therefore identified their impairment according to the World Health Organization's (WHO) classification of visual impairments. Their corrected binocular visual acuity is less than $3 / 10$ and above $1 / 10$, with a visual field of at least $20^{\circ}$ (category I of the WHO classification). They are schooled in the same specialized institute, and do not read Braille. They use computers with screen readers

on a daily basis. The study protocol followed the tenets of the Declaration of Helsinki of June 1964 (amended during the 64th General Assembly of the World Medical Association in October 2013). Experiments were carried out in the Normandie Lorraine Center. 
Design and experimental procedures

Participants were grouped in pairs. Each subject sits in front of a work-station including a laptop equipped with the Tactos software, an audio headset by which the subject can receive instructions, a computer mouse (whose software acceleration is identical and controlled for all the participants), and a set of tactile stimulators (a matrix of 16 piezoelectric pins adequately configured for each condition). A third computer manages the connexions and records the data.

The digital space in which the participants move is a horizontal one-dimensional line of 400 pixels which loops on itself (there are no edges or borders).

With the mouse, each participant moves a 2-pixel-long avatar, to which corresponds topologically a cursor, divided into four receptive fields (4 pixels long for each); the sensitivity of that cursor varies with the experimental conditions. Static objects of 2 pixels long are placed in the shared space. One of these objects can be detected by both participants (the "public" object), and the two others can be, respectively, detected only by the one and by the other ("private" objects) (Figure 3).

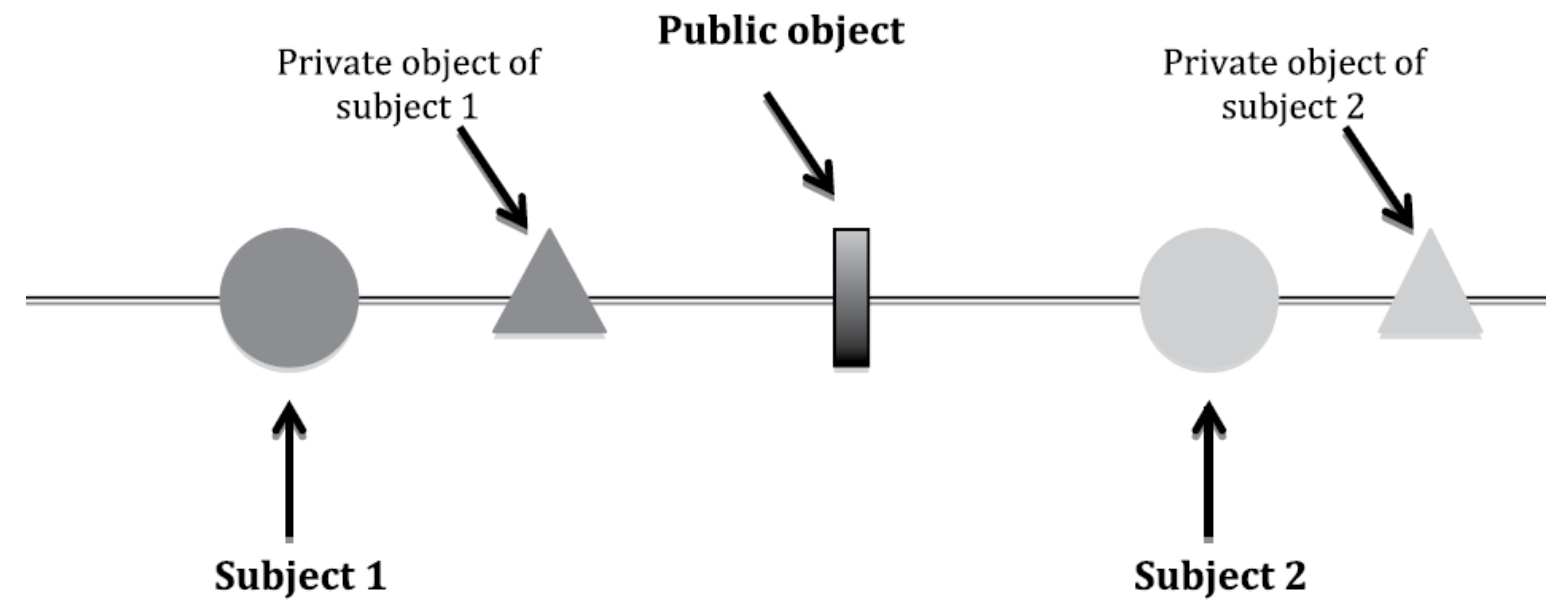

Figure 3 : Representation of the digital space

Two experimental conditions were retained from the study of Deschamps (2013) for the tactile feedback. In the "Differentiated" condition, when the cursor crosses at least one pixel of a static object (whether public or private), the four stimulators on the top line of the matrix are activated simultaneously, and when it crosses at least one pixel of the avatar moved by the partner, the four stimulators on the bottom line of matrix are activated simultaneously. With this configuration, the discrimination between the static objects and the avatar of the other is thus given to the participants via different sensory information.

In the "Parallelism" condition, the static objects and the avatar are not differentiated: they produce identical stimulations. However, when each receptor field crosses at least one pixel (from objects or from the avatar of the other), the tactile stimulators are activated by the simultaneous raising of one or more corresponding columns of the matrix of stimulators. Consequently, in addition to knowing their position with respect to the objects they encounter, the subjects can perceive the direction of the movement of the other subject when they cross the avatar. 


\section{Procedure}

The paired subjects met each other in a classroom of the institution. They were not allowed to speak during the experiment and they did not have access to the computer screens. Both were informed that they would embody two imaginary characters who have to rescue their village. Each pair passed the two conditions, in a random order. Each condition started with five trials of familiarization. The first of them enabled the participants to explore the space individually and to notice the activation of the tactile stimulators when they cross an object. Next, they were connected in a shared space and were successively assigned the roles of guide and follower. The follower could only detect the guide and had to follow her until they reached an audible target, which is heard only by the guide. The issue was for each subject to understand that the activation of the stimulators results from the encounter between her own avatar and the avatar of the other, and that it is necessary to collaborate in order to succeed in the task.

Then, the experimenter made sure that the subjects have understood the functioning of the device and the principle of the current condition, before engaging in the experimental phase, which is composed of $75 \mathrm{~s}$ trials. The participants had to interact and collaborate so as to click on the public object while avoiding the other objects. During each trial, they were informed that they could click during the last $15 \mathrm{~s}$ of the trial. Whatever the localization of their click, they received an auditory feedback telling them which source of stimulation they had clicked on (public object, private object, avatar of the other subject, empty). We may note that they did not receive any feedback if they click during the first minute of the trial, or if they did not click at all. During the course of each trial, the activity of the subjects was recorded.

At the end of the experiment, a semi-structured interview was proposed to the participants. The objective consisted in exploring what they felt during the experiment, the possible difficulties they encountered, and the strategies they deployed in order to solve the task. All interviews were conducted face to face and were inspired by the explicitation interview technique (Vermersch, 2011), in order to focus on the descriptive verbalization of lived emotion, sensation, and action during the task.

We hypothesized that the contextualization of the task would promote the collaboration of the adolescents, allowing them to click more often on the public object (correct response) than on any other source of stimulation. According to the results obtained by sighted adults in the study of Deschamps (2013), we also thought that collaboration would be facilitated by the tactile clues provided in the "Differentiated" condition. In other words, the adolescents would click more often on the public object in the "Differentiated" condition than in the "Parallelism" condition.

\section{Results}

Participants were required to find the public object and to click on it. They could click upon four distinct entities: the public object, their private object, the avatar of the other participant (when that participant was not on the public object), or upon empty space. They could also give no answer. For the analysis below, clicks on the public object were counted as "correct responses," all the other clicks were regrouped as "wrong responses," and no response were regrouped as "no response."

Since the distribution of our data did not fit a normal curve, we employed nonparametric statistics (Friedman and Wilcoxon tests).

The frequency of correct responses $(69.53 \%)$ was higher than the frequency of incorrect responses (33.85\%) and the frequency of no response $(0 \%)$. The difference 
between the frequency of correct responses and the frequency of incorrect responses was statistically significant $(p=.002)$. The adolescents thus clicked more frequently on the public object than on the other objects (Table 1).

Table I. Mean (standard deviation) and percentages according to the type of response $(N=12)$.

\begin{tabular}{lcl}
\hline & $M(S D)$ & $\%$ \\
\hline Correct responses & $11.12(1.65)$ & 69.53 \\
Wrong responses & $5.41(1.66)$ & 33.85 \\
No response & $0(0)$ & 0 \\
\hline
\end{tabular}

More specifically, the frequency of correct responses in the "Parallelism" condition is statistically higher than the frequency of correct responses in the "Differentiated" condition $(72.91 \%$ vs $59.37 \%, p=.002)$ (Table 2$)$.

Table 2. Mean (standard deviation) and percentages of responses according to the conditions $(N=12)$.

\begin{tabular}{llllll}
\hline & \multicolumn{2}{l}{ Differentiated condition } & & \multicolumn{2}{l}{ Parallelism condition } \\
\cline { 2 - 3 } & $M(S D)$ & & & $M(S D)$ & $\%$ \\
\hline Correct responses & $4.74(1.13)$ & 59.37 & & $5.83(1.69)$ & 72.91 \\
Wrong responses & $3.24(1.13)$ & 40.62 & & $2.16(1.69)$ & 27.08 \\
No response & $0(0)$ & 0 & & $0(0)$ & 0 \\
\hline
\end{tabular}

If we detail the results obtained in the "Parallelism" condition, we find that the participants obtained statistically more correct responses than incorrect responses (72.91\% vs $27.08 \%, p=.001)$. In contrast, in the "Differentiated" condition, while the adolescents clicked more frequently on the public object $(59.37 \%)$ than on the other objects $(40.62 \%)$, this difference is not statistically significant $(p=.17)$.

As regards to the analysis of semi-structured qualitative interviews, the emotional experience of the participants is associated with elements of the context: "I wanted to jump on the stomach of Perceptus," "Finding the house of the witch Bug, that was really hard," or yet again "I just had to follow Tac!" When questioned concerning their sensory experience, we observed that the subjects focused on the quantity of information provided by the tactile stimulators: "I preferred the house of the witch Bug ('Parallelism' condition) because there were more pins which lifted" and "I felt almost nothing with Perceptus ('Differentiated' condition)." Finally, the analysis of their experience of action revealed their difficulty in deploying actions relevant for reaching the goal: "In the beginning it was a bit hard, but after a bit you begin to understand and you can get by!"; "Sometimes I didn't really know what sort of object I was on." Several adolescents explained that they tried to implement strategies using what they were required to do during the "Guide-Follower" familiarization task ("I used the way we did with the owl and the toad," "I followed the other person").

\section{Discussion}

Here, we expected that the contextualization of the task would help the adolescents to collaborate, and we explored this process according to two configurations of tactile stimulation. 
We observed that the context of the environment and the feedbacks enabled subjects to succeed in the task, whatever the condition. The modification of the initial protocol seems to have improved the commitment of the participants in the task. Indeed, when they evoke their emotional experience, they refer to the elements that constitute the environment and to the characters. Similarly, and contrary to our previous study (Vallée et al., 2012), we did not observe an absence of clicks. This reinforces the idea that with this new environment, the adolescents better understood the task, and consequently, they were more involved to solve the task. Hence, a meaningful context and appropriate feedbacks upon their performance have actually promoted the collaboration between the adolescents (Stahl, 2006), which was not necessary for adults (Deschamps, 2013). Indeed, the perceptual activities of these subjects (the number and the duration of encounters between the avatars) are mostly organized around the shared object, and they recognize this object as being in common (Deschamps, 2013). When analyzing the correct responses according to the conditions, we observe that the adolescents obtained better results in the "Parallelism" condition. The success of the task seems to depend on the configuration of the tactile stimulations and the strategies they favor. In the

"Parallelism" condition, it is necessary to remain in contact with the avatar of the other subject and to regularly move one's own avatar (in the form of lateral oscillations) in order to distinguish the various sources of stimulation, thus favoring the mutual following of both participants. By contrast, in the "Differentiated" condition, the discrimination of the sources of stimulation (avatar/object) is given directly to the subjects; hence, they do not have to make this distinction by means of their own perceptual activity. The coordination of the perceptual activities thus takes place in the neighborhood of the static objects where the subjects stop in order to understand whether the other also perceives stimulation in relation to this object (Deschamps, 2013).

The analysis of the sensory experience also shows a preference for the "Parallelism" condition, where the participants think they have received a larger number of stimulations than in the "Differentiated" condition. This feeling could be simply induced by the successful following of the avatar of the other more than a "richer" stimulation per se. We may note here that the analysis of the experience of the action shows that the participants implement a strategy based on the principle of the familiarization trials, "Guide-Follower," a strategy which seemed to be facilitated in the "Parallelism" condition.

Thus, collaboration between visually impaired adolescents seems to be facilitated in the "Parallelism" condition, where they could follow each other. On the contrary, the sighted adults performed better in the "Differentiated" condition but they set up a strategy which does not involve permanently following the avatar of the other (Deschamps, 2013).

This difference calls for further investigation since it could be due to the visual status, to the age, and to the contextualization of the digital environment.

\section{Limitations and further research}

These initial results orient us in the conception of a pedagogical aid promoting collaboration between young subjects in a situation of visual impairment. Nevertheless, it seems essential to pursue this research and to refine our interpretations by analyzing the behavioral clues issuing from the recordings of the movements of the cursors. These analyses will be carried out using a model

from non-linear dynamic systems theory (Vallée, Rovira, Toba, \& Letellier, 2014). 
The second aim of this work being to enable sighted subjects to collaborate with visually impaired subjects; we will pursue this research with young sighted subjects.

\section{Conclusion}

This study brings to the fore that the context and feedback play an important role during collaboration between visually impaired adolescents in Intertact-like environments, and that the tactile feedbacks which promote the following of the avatar of the other lead to an improved collaboration.

These initial results orient us in the conception of an environment which can promote collaboration between visually impaired pupils, in the way that CSCL does for sighted pupils (loannidou et al., 2010).

\section{Acknowledgements}

The authors wish to thank the Normandie Lorraine Centre for their welcome.

\section{Declaration of Conflicting Interests}

The author(s) declared no potential conflicts of interest with respect to the research, authorship, and/or publication of this article.

\section{Funding}

The author(s) disclosed receipt of the following financial support for the research, authorship, and/or publication of this article: This study was partly supported by the ITOIP project (Picardy Region) and the large research network (GRR) from Upper Normandy "Persons in a situation of impairment: technical aids and human accompaniment."

\section{References}

Auvray, M., Lenay, C., \& Stewart, J. (2009). Perceptual interactions in a minimalist virtual environment. New Ideas in Psychology, 27, 32-47.

Capin, T. K., Pandzic, I. S., Thalmann, N. M., \& Thalmann, D. (1997, April). Realistic avatars and autonomous virtual humans in VLNET networked virtual environments. Proceedings of From Desktop to Webtop: Virtual Environments on the Internet, WWW and Networks, International Conference, 1-18, Bradford, UK.

Deschamps, L. (2013, January 21-25). Minimalist perceptual crossing: The case of triadic interactions. Seminaire PHITECO (Philosophie, Technologie, Cognition): From Perceptual Interactions to Extend Cognition, Université de Technologie de Compiègne, France.

Fiebich, A., \& Gallagher, S. (2013). Joint attention in joint action. Philosophical Psychology, 26, 571-587.

loannidou, A., Repenning, A., Webb, D., Keyser, D., Luhn, L., \& Daetwyler, C. (2010). Mr Vetro: A collective simulation for teaching health science. Intentional Journal of Computer-Supported Collaborative Learning, 5, 141-166.

Jansson, G., Juhasz, I., \& Cammilton, A. (2006). Reading virtual maps with a haptic mouse: Effects of some modifications of the tactile and audio-tactile information. British Journal of Visual Impairment, 2, 60-66. 
Resta, P., \& Laferrière, T. (2007). Technology in support of collaborative learning. Educational Psychology Review, 19, 65-83.

Rovira, K., \& Gapenne, O. (2009). Tactile classification on traditional and computerized media in three adolescents who are blind. Journal of Visual Impairment and Blindness, 103, 430-435.

Rovira, K., Gapenne, O., \& Vallée, A. (2014). Perceptual supplementation in the blind adolescent : individual strategies, perception and categorization of shapes. Enfance, 1, 89-106.

Searle, J. (1990). Collective intentions and actions. In P. Cohen, J. Morgan, \& M. Pollack (Eds.), Intentions in communication (pp. 401-415). Cambridge, MA: MIT Press.

Sribunruangrit, N., Marque, C., Lenay, C., Gapenne, O., \& Vanhoutte, C. (2002). Braille box: Analysis of the parallelism concept to access graphic information for blind people. Proceedings of the Second Joint Meeting of the IEEE Engineering in Medicine and Biology Society and the Biomedical Engineering Society (EMBSBMES), pp. 23-26, Houston, TX.

Sribunruangrit, N., Marque, C., Lenay, C., Gapenne, O., \& Vanhoutte, C. (2004). Speed-accuracy tradeoff during performance of a tracking task without visual feedback. IEEE Transactions on Neural Systems and Rehabilitation Engineering, 12, 131-139.

Stahl, G. (2006). Group cognition: Computer support for building collaborative knowledge. Cambridge, MA: MIT Press.

Vallée, A., Deschamps, L., \& Rovira, K. (2012). Supplementation device to blind children : interact through a haptic web. 54ième congrès national de la SFP 2012, pp. 253-255, Montpellier, France.

Vallée, A., Rovira, K., Toba, N. M., \& Letellier, C. (2014, June 23-26). Characterization of the dynamics underlying computer mediated communication of visually impaired and normal teenagers. ICCSA 2014, pp. 409-414, Normandie University, Le Havre, France.

Vermersch, P. (2011). Clarifying interview techniques. Paris: ESF. 